Hyades, of a factor near $50 \%$. It is very interesting to note that the helium to hydrogen ratio and oxygen to hydrogen ratio found by Faulkner and Aller in the Magellanic Clouds are in the same direction, and by what is a fairly comparable amount, i.e. $30 \%$.

Feast: Can Mr. Faulkner say whether a decrease in the adopted interstellar absorption will increase or decrease the $\mathrm{He} / \mathrm{H}$ ratio for 30 Doradus?

Faulkner: Yes, a decrease in the adopted interstellar absorption would decrease the $\mathrm{He} / \mathrm{H}$ ratio which I calculated.

Pagel: A comment on Dr. Arp's statement. Did he say that the Sun is deficient in metals, and if so, could he amplify this statement?

Arp : I judge the metal deficiency of the Sun relative to the Hyades by the three-colour $U, B, V$ photometry.

Pagel: The curve of growth doesn't demonstrate this.

Aller: The $\mathrm{He} / \mathrm{H}$ ratio can be estimated by comparing $\mathrm{HeI} \lambda 4471$ to $\mathrm{H} \gamma \lambda 4340$; the effects of interstellar absorption cannot be important in this case.

Gascoigne: How far are the abundance results dependent on assumptions on the uniformity of the regions studied?

Faulkner: This will vary from nebula to nebula. In the case of the 30 Doradus nebula, the region studied is a loop which appears very uniform, and is considerably brighter than surrounding regions. Thus virtually all the light received is coming from this uniform region, and the effect will be small.

Gascoigne: If the structure were patchy as the Orion nebula is believed to be, in which direction would the abundance be affected?

Aller: This depends on the element considered.

Faulkner : The emission per unit volume in a HeI recombination line is proportional to the product of the number densities of ionized hydrogen and singly ionized helium, $N\left(\mathrm{H}^{+}\right) \cdot N\left(\mathrm{He}^{+}\right)$. Similarly the emission per unit volume in a Balmer line is proportional to $\left[N\left(\mathrm{H}^{+}\right)\right]^{2}$. Thus, provided the ratio $N\left(\mathrm{He}^{+}\right) / N\left(\mathrm{H}^{+}\right)$remains constant throughout the density fluctuations, the relative intensities of the helium and hydrogen recombination lines will be unchanged, and the density fluctuations will have no effect on the calculated abundance of helium.

For oxygen the situation is far more complicated, since here we are dealing with forbidden lines, and the density also plays a part in determining the balance between collisional and radiative de-excitation of the metastable levels.

\title{
65. PLANETARY NEBULAE AND WOLF-RAYET STARS IN THE MAGELLANIC CLOUDS
}

\author{
B. E. WesterLund \\ Mount Stromlo Observatory
}

\section{The Planetary Nebulae}

Objects in the Small Magellanic Cloud classified by Lindsay (1961) as planetary nebulae or probable planetary nebulae have been studied in detail on large-scale photographs (Henize and Westerlund 1963). Of Lindsay's 50 objects, 11 are clearly resolved, 2 are probably resolved, 12 show stellar images, 13 are below the limit of the plates, 11 (fairly faint) are outside the photographed regions, and 1 is of uncertain identification. The masses of the resolved nebulae lie between 2 and 33 solar masses. They are therefore classified as small diffuse nebulae; it appears unlikely that the mass of a planetary nebula can exceed a few tenths of a solar mass. 
It is probable that most of the unresolved objects are true planetary nebulae. None of them lies above $M_{p g}=-3 \cdot 0$ (distance modulus of the Clouds, $m-M=$ $18.7 \mathrm{mag}$ ), and it is concluded that this value gives the upper limit to the luminosity of planetary nebulae.

The upper limit of the total number of planetary nebulae in the Small Magellanic Cloud can be determined, if we assume that all of Lindsay's objects with $M_{p g}>-3 \cdot 0$ are true planetary nebulae, that these nebulae due to their proximity to the maximum magnitude are all in or just past the optically thick stage, and that this phase in their evolution occupies one-tenth of the lifetime of the nebulae (O'Dell 1962, Fig. 4). Under these assumptions we find a total of approximately 300 planetaries in the Small Cloud. This figure may be compared with O'Dell's estimate of $4.8 \times 10^{4}$ planetaries in the Galaxy. It appears that the number of planetaries per unit mass in the two galaxies is in good agreement.

TABLE 1

PLANETARY NEBULAE IN THE LARGE MAGELLANIC CLOUD

$$
m-M=18 \cdot 7
$$

Total number of planetary nebulae: 42

Number within $2^{\circ}$ of centroid: 21

For 10 brightest objects: $\overline{B n}=15 \cdot 75 \pm 0 \cdot 02$ (s.e.)

$$
\overline{M_{B}^{n}}=-3 \cdot 0
$$

For all objects:

$$
\begin{aligned}
\overline{B^{n}} & =16 \cdot 50 \pm 0 \cdot 10 \\
\overline{M_{B}^{n}} & =-2 \cdot 2 \\
\overline{B-R} & =1 \cdot 48 \pm 0 \cdot 08
\end{aligned}
$$

Number of objects with central star observed: 18.

$$
\begin{aligned}
\overline{B^{s}} & =16 \cdot 93 \pm 0 \cdot 15 \\
\overline{M_{B}^{8}} & =-1 \cdot 8 \\
\overline{B^{n}} & =16 \cdot 66 \pm 0 \cdot 17 \\
\overline{M_{B}^{n}} & =-2 \cdot 0 \\
\overline{B^{8}-B^{n}} & =+0 \cdot 27 \pm 0 \cdot 14
\end{aligned}
$$

A search for planetary nebulae in the Large Cloud was carried out a few years ago by Westerlund and Rodgers (1959). The survey has been continued, the bright objects have been studied on large-scale photographs, and photometry of the nebulae and, in some cases, of the central stars has been carried out (Westerlund and Lindsey Smith, unpublished data). The results are summarized in Table 1 . It is again established that the upper limit to the luminosity of planetary nebulae is $M_{B}=-3.0 \mathrm{mag}$.

The blue $(B)$ magnitudes of the central stars of the planetary nebulae have been determined from their visual magnitudes $(V)$ under the assumption of a colour of $B-V=-0 \cdot 2$ mag. The derived mean magnitude difference between stars and nebulae, $\overline{B^{s}-B^{n}}=+0.27 \mathrm{mag}$, is in good agreement with O'Dell's results for the most luminous galactic planetary nebulae. It may be taken as another supporting proof of Shklovsky's model of the evolution of the planetary nebulae (Shklovsky 1956). 
The total number of planetary nebulae in the Large Cloud is about 400 under the same assumptions as above. This gives a number per unit mass in the Large Cloud which is only one-third of that found above for the Small Cloud and for the Galaxy.

\section{The Wolf-Rayet Stars}

A few years ago the Large Cloud was searched for Wolf-Rayet stars by Westerlund and Rodgers (1959). The survey has been continued and photometry, in blue $(B)$, visual $(V)$, and red $(R)$ light, has now been carried out (Westerlund and Lindsey Smith, unpublished data). Using as standards stars classified by Feast, Thackeray, and Wesselink (1960), we have attempted to divide our material into WC, WN, and $\mathrm{W}+\mathrm{O}$ stars. We have also divided the stars into field objects, objects in associations, and members of the 30 Doradus complex. A Wolf-Rayet star has been assumed

TABLE 2

WOLF-RAYET STARS IN THE LARGE MAGELLANIC CLOUD

\begin{tabular}{|c|c|c|c|c|}
\hline & $n$ & $\bar{V}$ & $\overline{B-V}$ & $\overline{V-R}$ \\
\hline Identified objects: & 53 & $13 \cdot 32 \pm 0 \cdot 19$ & $-0 \cdot 17 \pm 0 \cdot 03$ & $-0.08 \pm 0.05$ \\
\hline WC & 5 & $13 \cdot 12 \pm 0 \cdot 29$ & $+0.06 \pm 0.07$ & $-0 \cdot 59 \pm 0 \cdot 06$ \\
\hline WN & 23 & $13 \cdot 82 \pm 0 \cdot 21$ & $-0 \cdot 19 \pm 0 \cdot 03$ & $-0.01 \pm 0.05$ \\
\hline $\mathbf{W}+\mathrm{O}$ & 9 & $11 \cdot 23 \pm 0 \cdot 34$ & $-0 \cdot 18 \pm 0 \cdot 10$ & $-0.01 \pm 0.07$ \\
\hline Association objects: & 23 & $12 \cdot 63 \pm 0 \cdot 29$ & & \\
\hline Field objects: & 24 & $14 \cdot 24 \pm 0 \cdot 16$ & & \\
\hline 30 Doradus objects: & 6 & $12 \cdot 02 \pm 0 \cdot 28$ & & \\
\hline
\end{tabular}

to be a member of an association if its distance from the centre of the association is less than two times the radius. It has been accepted as a member of the 30 Doradus complex only if it is obviously in the nebula. To the six objects identified by us in the outer parts of 30 Doradus, five more may be added from the catalogue by Feast and co-workers.

Table 2 summarizes the results of our survey. It is of particular interest to note:

(1) The difference in colour, $V-R$, between the WC and the WN stars. This difference appears to be caused by the strong emission of OV (5590 $\AA$ ), CIII (5696 $\AA$ ), and CIV (5810 $\AA$ ) in the visual region of WC stars, whereas the WN stars generally only show HeI (5876 $\AA$ ) and HeII (5411 $\AA$ ) there. A similar effect is found in $B-V$. It appears possible to assign Wolf-Rayet stars WN or WC type from their $(V-R)$ colours.

(2) The identified $\mathrm{W}+\mathrm{O}$ stars are on an average more than 2 mag brighter than the stars where no binary characteristics have been noted. However, it must be pointed out that selection effects are possible. An object can be identified as of class $\mathrm{W}+\mathrm{O}$ only if the continuum can be seen. 
It may also be added that all the identified $\mathrm{W}+\mathrm{O}$ stars are members of associations.

(3) The members of associations are on the average $1 \cdot 6$ mag more luminous than the field objects and the members of the 30 Doradus complex are still $0.6 \mathrm{mag}$ more luminous. It appears likely that this is an indication of differences in age. The more luminous objects are found in the younger stellar groups. It may be noted as a curiosity that among the larger stellar associations, only Shapley's constellation I (NGC 1929-34-35-36-37) has so far no identified Wolf-Rayet star. In this aspect constellation I reminds us of the Orion nebula where no Wolf-Rayet star has yet been found (Roberts 1962).

The total number of known Wolf-Rayet stars is 58 in the Large Cloud, one or possibly two in the Small Cloud (Buscombe, Gascoigne, and de Vaucouleurs 1955), and 123 in the Galaxy (Roberts 1962). The agreement in numbers per unit mass

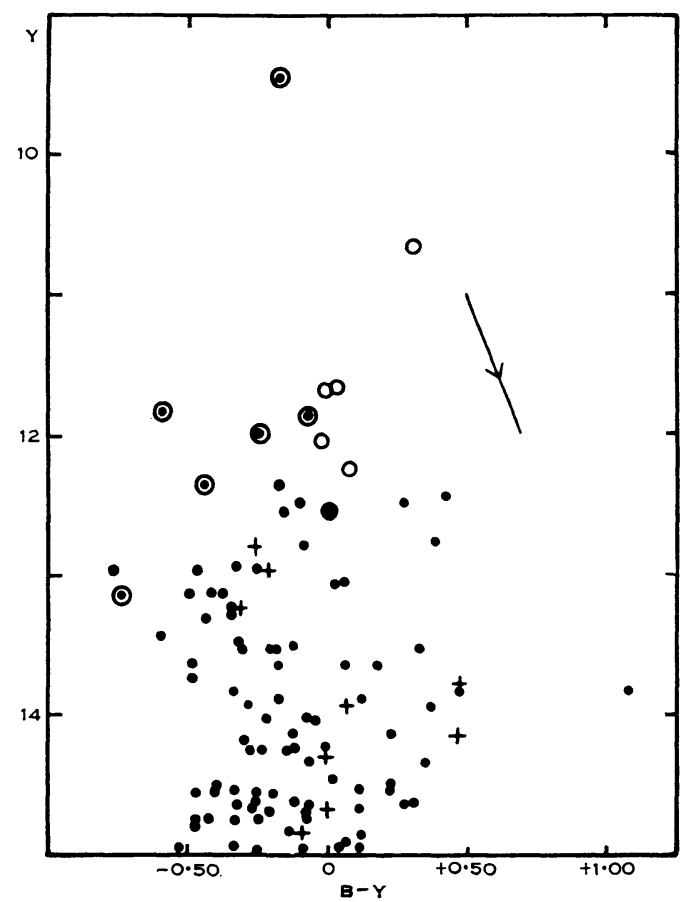

Fig. 1.-The colour-magnitude array for the central region of 30 Doradus. $y$ and $(b-y)$ are in Strömgren's four-colour system. Open circles represent supergiants, dots in circles Wolf-Rayet stars, and the large dot an 08 star. Plus signs denote stars in a small cluster to the north-west of the nucleus. The arrow indicates the reddening path.

is again better between the Small Cloud and the Galaxy than between the Large Cloud and the others. Obviously, some corrections for the incompleteness of the observed numbers are desirable. In any case, the data for planetary nebulae and Wolf-Rayet stars strengthen the impression that the Large Cloud has a predominantly vounger population than the other two galaxies. 


\section{The Wolf-Rayet Stars in the 30 Doradus Complex}

The central region of 30 Doradus contains six Wolf-Rayet stars of which one only is included in Table 2. The five others, embedded in bright nebulosity, have been classified by Feast et al. from slit spectra.

Photometry of all the stars brighter than $V=15$ mag in the central region of 30 Doradus has recently been carried out (Westerlund, unpublished data) in Strömgren's four-colour system using filters similar to those described by Crawford (1961). Figure 1 shows the derived preliminary colour-magnitude diagram. Identified supergiants are denoted as open circles, Wolf-Rayet stars as dots in circles, and an identified 08 star as a large dot. The classification is, in all cases, by Feast and coworkers.

We note that most of the Wolf-Rayet stars fall at the turn-off point of the main sequence in agreement with previous observations of other associations in the Large Cloud (Westerlund 1961). The nucleus of 30 Doradus, classified $\mathrm{O}+\mathrm{WN}$ at Radcliffe, forms an obvious exception with a magnitude of $y=9.5 \mathrm{mag}$. However, this star is suspected of being still more composite.

It may be of interest to note here that the total mass of the stars in the central region of 30 Doradus, inside $22 \cdot 4$ square minutes of arc (or about 80 by $80 \mathrm{pc}$ ), is of the order of $2 \times 10^{4}$ solar masses under the same assumptions as used previously (Westerlund 1961). The stellar density in the central cluster itself is of the order of one solar mass per cubic parsec.

\section{References}

Buscombe, W., Gascoigne, S. C. B., and de Vaucouleurs, G. (1955).-Aust. J. Sci. 17 : No. 3, Suppl.

Crawford, D. L. (1961).-A.J. 66: 281.

Feast, M. W., Thackeray, A. D., and Wesselink, A. J. (1960).-M.N. $121: 337-85$.

Henize, K. G., and Westerlund, B. E. (1963).-Ap. J. 137 : 747-57.

LiNDSAY, E. M. (1961).-A.J. $66: 169-85$.

O'DeLL, C. R. (1962).-A p. J. 135 : 371-84.

ROBERTS, M. S. (1962).-A.J. 67: 79-85.

SHKLovsky, I. S. (1956).-Astr. Zhurn. USSR 33: 315-29.

Westerlund, B. E. (1961).-U Upsala Obs. Ann. 5 : No. 1.

Westerlund, B. E., and Rodgers, A. W. (1959).-Observatory 79: 132-4.

\section{Discussion}

Thackeray: Some time ago Dr. Koelbloed working at the Radcliffe Observatory carried out a brief survey of fields in the SMC for planetaries. He used a technique apparently very similar to Dr. Westerlund's with 103aJ plates and filter to isolate $N_{1} N_{2}$. His absolute magnitudes ran fainter than Lindsay's. Has your survey covered some of Koelbloed's objects or have you not had access to the detailed results?

Westerlund: Koelbloed's objects agree in distribution with Lindsay's. There is no way of identifying his individual objects. Therefore we used Lindsay's catalogue for our observations.

Buscombe: Can the most luminous stars $\left(M_{V} \sim-7\right)$ you have mentioned be compared with the Of stars which dominate the youngest open clusters and associations in the Milky Way?

Westerlund: It is difficult to distinguish, at least in the Magellanic Clouds, between WR and Of stars on objective prism plates. 
Feast: In answer to Dr. Buscombe I think it would be generally admitted that Of stars are probably an example of mild WR activity.

Buscombe: Less luminous, however.

Feast: We don't know the lower limit to WR stars in the Galaxy.

Buscombe: Existing plate material should be surveyed for novae that have recently been overlooked in the Magellanic Clouds.

Ambartsumian: What is the faint limit of your survey of WR stars especially in and around the region of 30 Doradus?

Westerlund: Inside 30 Doradus my identification depends entirely on Thackeray's results as we cannot identify WR stars, because of the emission nebulosity on objective prism plates. Outside, the faintest is about 15th magnitude.

Aller: Magnitudes of planetaries secured with a broad band-pass filter are difficult to interpret in terms of monochromatic fluxes. With regard to $\mathrm{B}$ magnitudes, one notes that the ratio of the green nebula [OIII] lines to $\mathbf{H} \beta$ ranges from considerably less than 1 (NGC 40) to around 20.

Westerlund: I have discussed this point with Baade and Minkowski. They agreed that one must use B magnitudes for statistical purposes.

Eggen: Is it not possible that there is a difference for WR stars inside and outside 30 Doradus?

Westerlund: It is possible but I do not think that the effect is seen on slit spectra of the objects.

\title{
66. SUPERGIANTS IN THE MAGELLANIC CLOUDS AND IN THE GALAXY
}

\author{
Th. WaLraven and J. H. Walraven \\ Mount Stromlo Observatory
}

\section{Introduction}

With the five-colour photometer of the Leiden Southern Station (system $V, B, L, U, W$ ) stars can be classified according to luminosity (Th. Walraven and J. H. Walraven 1960).

The method was used in a search for member stars of the Magellanic Clouds, which are supergiants and can be easily distinguished from the galactic foreground stars, which are dwarfs. In addition, already recognized members from the list of Feast, Thackeray, and Wesselink (1960), and O-B stars from the HDE catalogue were observed. In total about 600 stars were inspected, of which roughly $50 \%$ turned out to be members. The result of this investigation was read in a paper at the IAU Symposium XI, at Berkeley, but it was not published in detail; we decided to complete the work with the investigation of many more bright galactic stars of different spectral type and luminosity. This program included approximately 300 stars.

In this paper we give only some results of a comparison of the Magellanic stars with the galactic supergiants and the detailed results and catalogue of stars will be published in the Bulletin of the Astronomical Institutes of the Netherlands. 